\title{
Diagnostic accuracy of double inversion recovery (DIR) in detection of cortical gray matter lesions in patients with MS
}

\author{
Mahmoud M. Higazi ${ }^{1}$, Hosny Sayed Abd El Ghany ${ }^{1}$, Alaa Wagih Fathy' ${ }^{1}$, Muhammad Mamdouh Ismail ${ }^{2}$ and \\ Manal F. Abu Samra ${ }^{1 *}$
}

\begin{abstract}
Background: Conventional imaging techniques have a low sensitivity for detection of cortical and deep grey matter lesions in MS which hinder accurate assessment of the total lesion burden. Aim of this work was to assess the diagnostic accuracy of double inversion recovery (DIR) sequence in the detection of cortical grey matter lesions in MS patients.

Results: Forty MS patients were prospectively included in this study. Imaging was performed using Philips Ingenia 1.5 T device. The sensitivity, specificity, PPV, NPV and accuracy of DIR sequence in detection of cortical grey matter lesions were $60 \%, 100 \%, 100 \%, 55.6 \%$ and $73.3 \%$, respectively. The sensitivity, specificity, positive and negative predictive values as well as accuracy of Flair sequence were $50 \%, 100 \%, 100 \%, 50 \%$ and $66.7 \%$, respectively. The sensitivity, specificity, positive and negative predictive values as well as accuracy of T2 sequence in the detection of cortical grey matter lesions were $22.5 \%, 100 \%, 100 \%, 39.2 \%$ and $48.3 \%$, respectively.
\end{abstract}

Conclusions: Detection rate of cortical gray matter lesions was significantly higher on DIR sequence than on T2 and Flair sequences.

Keywords: MS, MRI, DIR versus FLAIR sequences

\section{Background}

Grey matter brain damage is common in multiple sclerosis (MS) which proved pathologically in an increasing manner, so changes the classic concept of MS as a pure white matter disease and has led to incorporation of cortical grey matter lesions in the recent diagnostic criteria for MS. Also, increase the interest in the role of normal appearing grey matter damage in determining cognition and disability [1].

Changes in the cerebral cortex have been associated with the progression of disability in MS patients, so cortical lesions are significantly more evident in patients with

\footnotetext{
*Correspondence: manal_abusamra@ymail.com

1 Department of Radiology, Faculty of Medicine, Minia University, Minya, Egypt

Full list of author information is available at the end of the article
}

more pronounced brain tissue damage [2]. Grey matter lesions not only correlate with physical disability but also with the level of cognitive impairment which develop in MS patients [3].

Conventional imaging techniques have a low sensitivity for detection of cortical and deep grey matter lesions in MS which hinder accurate assessment of the total lesion burden [1].

Newer MR imaging sequences such as double inversion recovery (DIR) is $1.5-5$ times more sensitive than conventional MRI in detection of grey matter lesions especially cortical lesions [4].

Double inversion recovery consists of two inversion pluses applied to suppress the signal from two tissues simultaneously. In the brain DIR is used mainly to selectively image the grey matter by nulling the signal from 
white matter and CSF [5]. So it provides higher image contrast between lesions and the normal appearing grey matter compared to other sequences due to differences in the relaxation times between grey matter and CSF with subsequent detection of more lesions' number [6].

In MS patients, DIR sequence has significantly increase the detection of cortical lesions including both intra-cortical lesions (ICLs, which are confined to cortical ribbon without involving underlying subcortical white matter) and particularly leukocortical lesions (LCLs, which affect both deep grey matter and adjacent white matter) which have the highest detection rate among cortical lesions [7].

Intra-cortical lesions are classified according to morphologic features into 4 types round or ovoid (most common) with clear margins, worm shaped following one or more gyri, wedge shaped and clusters of micro-granular lesions (infrequent) [7].

Double inversion recovery shows that cortical lesions are significantly more frequent and numerous at late stage of MS but can be demonstrated in early phases of the disease even in patients with a first attack [7]. Double inversion recovery is a potential imaging biomarker for cognitive deficits [3]. Also, by DIR sequence cortical lesions have been detected in all MS clinical phenotypes including CIS patients [7].

Double inversion recovery is currently considered the best wide scale application pulse sequence that offers high sensitivity, specificity and accuracy for the detection of grey matter lesions. It is an innovative MRI sequence constitutes a significant step forward in the demonstration of deep grey matter involvement and focal cortical pathologic conditions so it is useful for clinical purposes as a supplement to or replacement for standard FLAIR and $\mathrm{T} 2$ sequences.

Aim of the work is to evaluate the diagnostic accuracy of DIR in detection of cortical gray matter lesions in patients with MS.

\section{Methods (group I)}

In a prospective study, 40 patients clinically diagnosed to have MS according to 2017 Mc-Donald criteria were included. They were diagnosed in neuropsychiatry department, and they were referred to department of radio-diagnosis, Faculty of Medicine. The study was done between December 2017 and February 2020.

Inclusion criteria:

Patients diagnosed with MS (clinical and laboratory).

Age between 18 and 50 years.

Expanded disability status scale (EDSS) score from 1 to 6.

Exclusion criteria:
The patients who were contraindicated for MRI examination as those had artificial cardiac pacemaker or metallic prosthesis.

Patients with severe claustrophobia.

Patients with age above 50 years old or less than 18 years.

Patients with concomitant neurological disease in conjunction with MS.

Patient with prior diagnosis of cardiovascular morbidity.

All patients were subjected to:

I) Clinical assessment:

II) Full history taking including name, sex, age and symptoms as well as duration of the disease.

III) Neurological assessment: Evaluation of disability using EDSS score as well as assessment of the course of the disease in our neuropsychiatry department.

IV) Imaging study:

\section{Brain MRI}

Brain MRI examination was performed on $1.5 \mathrm{~T}$ MRI system (Philips Ingenia). All patients were imaged in the supine position using head coil. The MRI protocol includes the following:

2D Axial T2 weighted images utilizing the following parameters: Repetition time (TR) of $4000 \mathrm{~ms}$, echo time (TE) of $98 \mathrm{~ms}$, slice thickness of $5 \mathrm{~mm}$, number of signal averages (NSA) $=1$ matrix $512 \times 512$, gap $1-2 \mathrm{~mm}$, flip angle $=90^{\circ}$ and field of view $(\mathrm{FOV})=230 \mathrm{~mm}$.

2D Axial and sagittal Flair images utilizing the following parameters: Repetition time (TR) of $6000 \mathrm{~ms}$, echo time (TE) of $120 \mathrm{~ms}$, slice thickness of $5 \mathrm{~mm}$, (NSA) 2, matrix $512 \times 512$, gap $1-2 \mathrm{~mm}$, flip angle $=90^{\circ}$ and FOV $=230 \mathrm{~mm}$.

Axial double inversion recovery (DIR) MRI sequence utilizing the following parameters: Repetition time (TR) $2400 \mathrm{~ms}$, echo time (TE) of $25 \mathrm{~ms}$, slice thickness of $3 \mathrm{~mm}$ and FOV $=220 \mathrm{~mm}$.

\section{Healthy controls (group II)}

Twenty healthy and age matched volunteers were examined in the analysis for comparison. They had no contraindication for MRI examination as well as no history for any neurological disease. They were subjected to the following: 2D axial Flair sequence, $2 \mathrm{D}$ axial T2, 2D axial DIR with the same parameters as our patients. 


\section{Image analysis for patients and control group}

Image interpretation was performed on communication system workstation using Philips ISP (Intell space portal V. 9) and then analyzed as follows:

Assessment of the distribution of MS plaques in different grey matter sites of the brain among 40 MS patients (including leukocortical, intracortical regions as well as deep grey matter region of the brain).

Assessment of the number of cortical grey matter lesions (including leukocortical and intracortical lesions) in different pulse sequences including T2, Flair and DIR. Leukocortical lesions were determined as lesions involve both cortex and juxtacortical white matter. Intracortical lesions were defined as focal lesions completely lie within the cortex and not affecting white matter.

Differentiation between juxtacortical lesions (which are white matter lesions touching cortex with no intervening white matter) and leukocortical lesions.

\section{Results}

The study was conducted in the department of Radiology, at the time period from December 2017 to February 2020. All patients were diagnosed in neuropsychiatry department, and they were referred to our department of radio-diagnosis for MRI examination.

\section{Demographics and clinical data}

Demographic and clinical variables in 40 multiple sclerosis (MS) patients and 20 controls who were included in the study are shown in the following Tables and Figs. 1, 2, 3, 4 and 5.

The included 40 patients were $(87.5 \%$ female and $12.5 \%$ were male) diagnosed as MS according to McDonald criteria 2017. Range of the age was (20-47) years and mean age of all patients was $(29.2 \pm 7.1)$ years (Table 1).

For the control group 20 healthy volunteers were examined in the analysis for comparison. All were female, age range was (24-48) years and mean age was $(32.75 \pm 9.23)$ years (Table 2$)$.

\section{Conventional MRI criteria}

As regarding distribution of MS plaques in different anatomical locations of the brain among 40 patients revealed that 22 patients (55\%) had leukocortical plaques while 10 patients (25\%) had intra-cortical plaques (Table 3 ).
A statistically significant difference was found between different MRI sequences (DIR, T2 and Flair) as regarding number of cortical grey mater lesions among studied MS patients $(P<0.001)$. In the present study, 254 cortical lesions were detected with DIR sequence and 214 lesions with Flair sequence while only 72 lesions were detected with T2 sequence.

DIR sequence demonstrated significantly more cortical grey matter lesions compared to Flair and T2 sequences (Table 4).

As regarding cortical grey matter lesions, there was statistically significant strong agreement between DIR and Flair $(K=0.8, P<0.001)$ and fair significant agreement between DIR and T2 $(K=0.32, P=0.005)$ as well as moderate significant agreement between Flair and T2 $(K=0.52, P<0.001)$ (Table 5).

It was found that there was statistically significant strong positive correlation between number of cortical grey matter lesions in different MRI pulse sequences (T2, Flair and DIR) versus EDSS. However, DIR sequence showed the highest significant positive correlation (Tables 6, 7).

ROC curve analysis shows significant diagnostic performance for detection of cortical grey matter lesions with DIR sequence $(A U C=0.8)$ and fair significant diagnostic performance with Flair sequence $(\mathrm{AUC}=0.75)$ while poor insignificant diagnostic performance was observed by $\mathrm{T} 2$ sequence $(\mathrm{AUC}=0.61$ ). So diagnostic performance of DIR sequence in prediction of cortical grey matter lesions was more than Flair and T2 sequences. The sensitivity, specificity, PPV, NPV and accuracy of DIR sequence in detection of cortical grey matter lesions were $60 \%, 100 \%, 100 \%, 55.6 \%$ and $73.3 \%$, respectively. The sensitivity, specificity, positive and negative predictive values as well as accuracy of Flair sequence were 50\%, 100\%, $100 \%, 50 \%$ and $66.7 \%$, respectively. The sensitivity, specificity, positive and negative predictive values as well as accuracy of T2 sequence in the detection of cortical grey matter lesions were $22.5 \%, 100 \%, 100 \%, 39.2 \%$ and $48.3 \%$, respectively (Table 8 ).

\section{Discussion}

Multiple sclerosis is chronic inflammatory, demyelinating and neurodegenerative disease of CNS. It is the second most common cause of disability after trauma in young adults. Magnetic resonance imaging (MRI) is an important non clinical tool for MS diagnosis. Great efforts have been dedicated in the current years to overcome conventional MRI limitations by the use of non-conventional MR metrics to provide detailed pathological information 


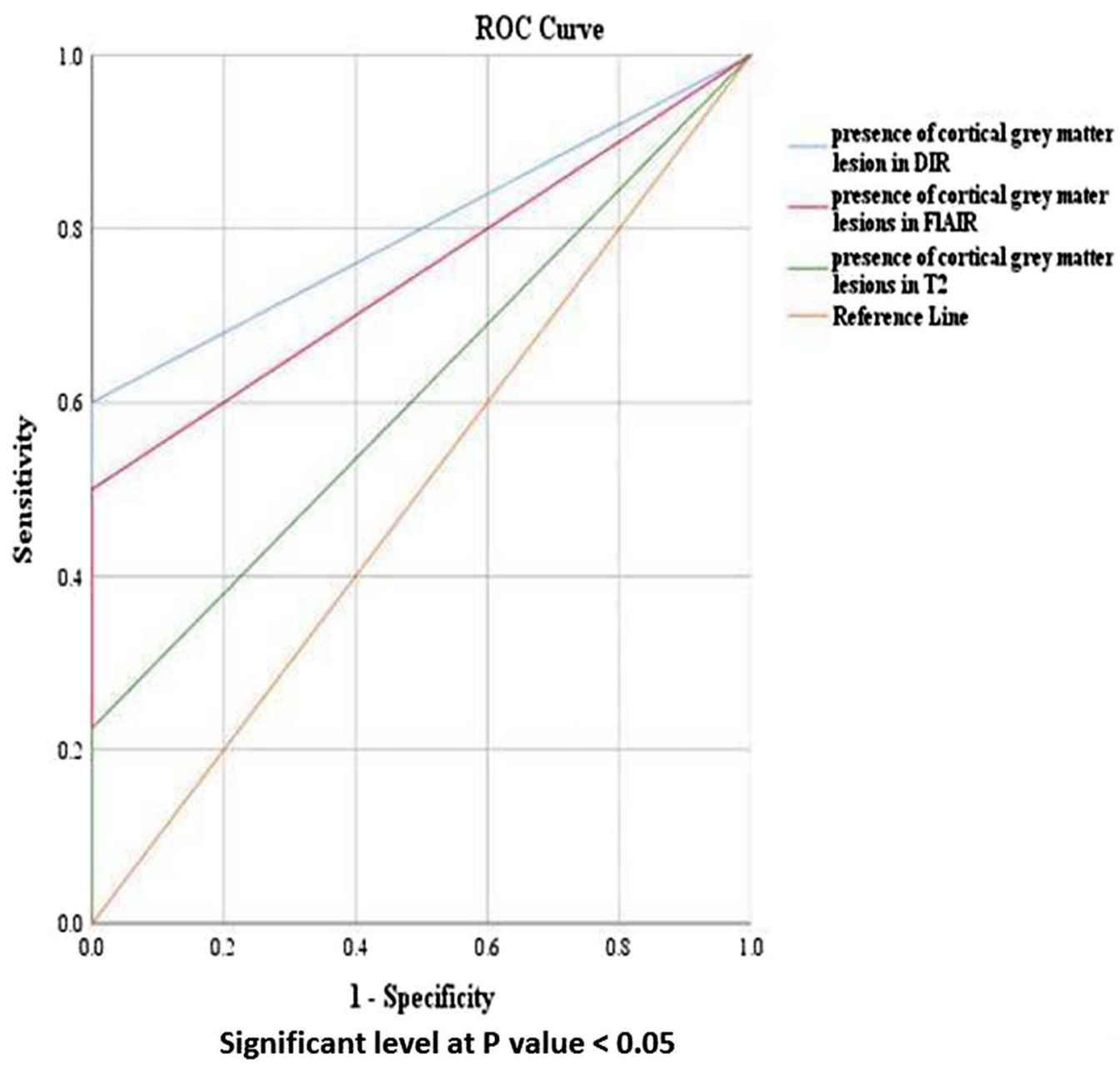

Fig. 1 ROC curve analysis for the prediction of cortical grey matter lesions using different MRI sequences (DIR, Flair and T2) (control group is the reference group)

related to the mechanisms of MS via different imaging techniques to reverse the neurodegenerative processes. Some of these metrics include double inversion recovery.

In this study 40 patients diagnosed with MS according to McDonald criteria 2017 were included and 20 healthy controls were examined in the study for comparison. None of the controls in our study reported to have any neurological conditions and their FLAIR images were judged to be normal. Double inversion recovery (DIR) is an important sequence and the advocacy for it came at a time when the assessment of grey matter lesions by conventional MRI is limited and not optimal because of poor contrast resolution between normal appearing grey matter and plaques, partial volume effects of CSF surrounding the cortex and due to low degree of inflammation. Recognition of grey matter involvement in MS has led to the incorporation of cortical/juxta-cortical lesions in 2017 revised Mc-Donald criteria for the diagnosis of MS. Double inversion recovery increases the sensitivity for detecting cortical lesions via suppressing signal from both CSF and white matter thus providing a remarkable delineation between grey matter and white matter as well as facilitates visualization of cortical grey matter lesions [8].

In this study, there was a good significant diagnostic performance of detection of cortical grey matter lesions with DIR sequence (AUC $=0.8$ ) in comparison with the Flair and T2 sequences. Detection rate of cortical lesions was significantly higher on DIR sequence than on T2 and Flair sequences. These findings were in agreement with Shilpa et al., Hamed et al. and Abidi et al. [9-11]. 


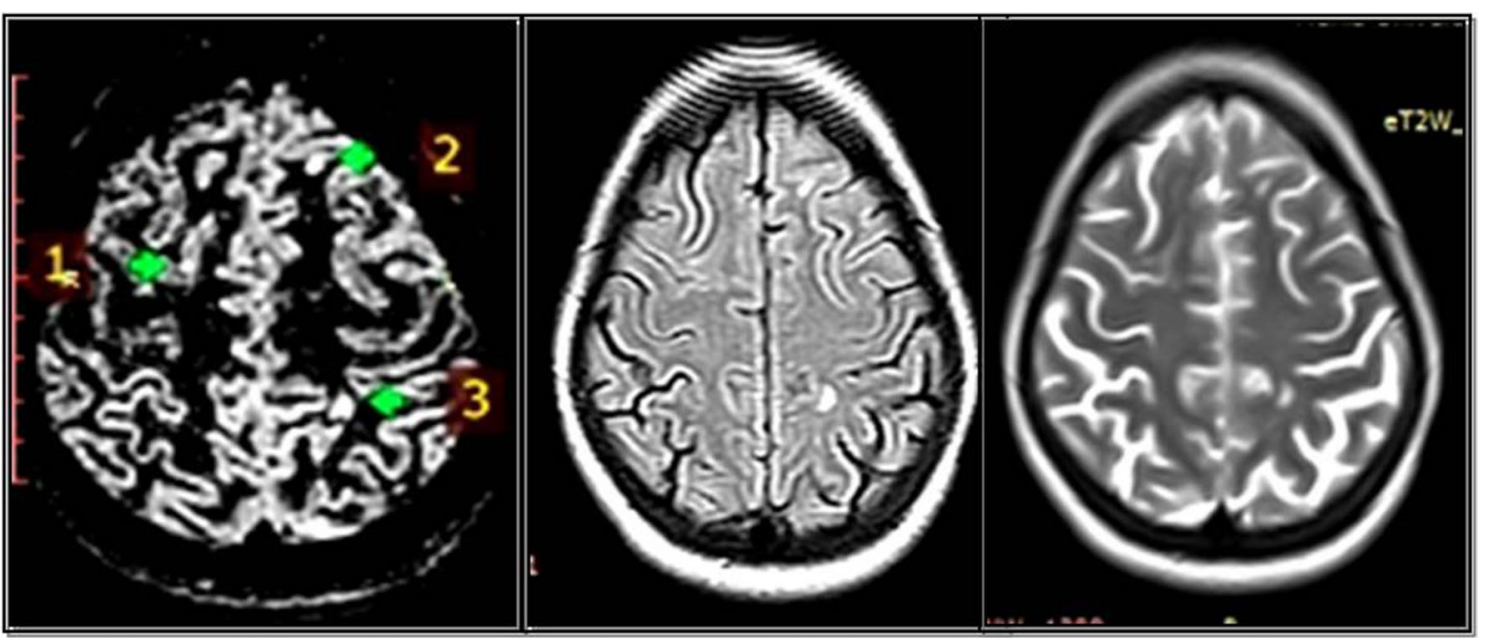

Fig. 2 Female 30-years-old with history of RRMS since 6 months presented by numbness and headache, EDSS $=2$. A-C (Axial DIR, Flair and T2 sequences, respectively) show multiple MS plaques more obvious with more localization on DIR sequence. ( 1 =intracortical, $2=$ juxtacortical and $3=$ WM lesion)

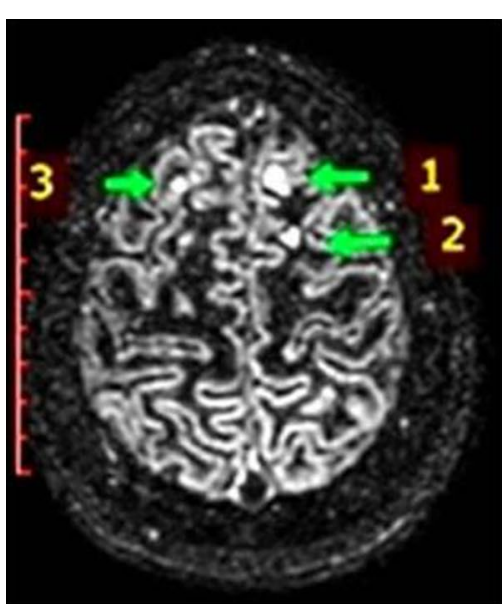

A

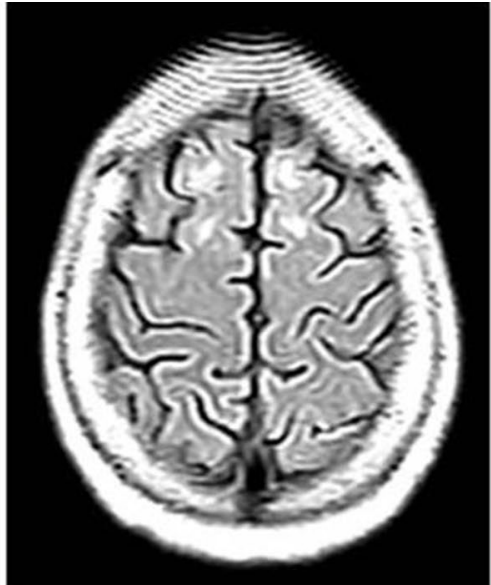

B

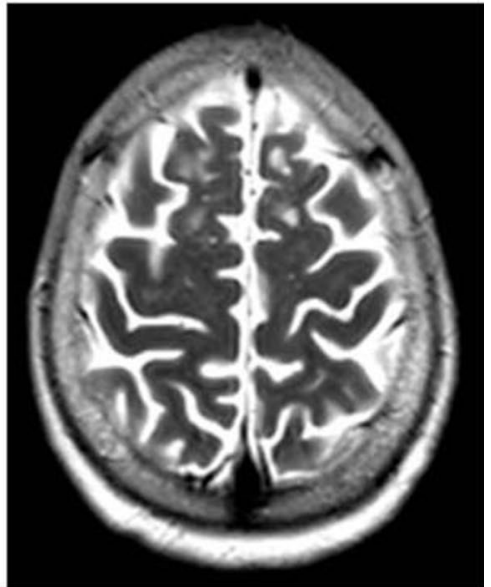

C

Fig. 3 Female patient 30-years-old with history of RRMS since 4 years presented by weakness, headache and visual disorder, EDSS $=4.5$. A-C (Axial DIR, Flair and T2 sequences, respectively) show MS plaques noted at different anatomical locations more obvious on DIR sequence. ( 1 = leukocortical lesion, 2 =WM lesion and $3=$ intra-cortical lesion)

In this study as regarding detection of cortical lesions, statistically significant strong agreement was found between DIR and Flair sequences $(K=0.8, P<0.001)$ while fair agreement was found between DIR and T2 sequences $(K=0.32, P=0.005)$.

There was positive strong correlation between number of cortical grey matter lesions in different MRI pulse sequences (T2, Flair and DIR) and EDSS score with DIR sequence showed the highest significant positive correlation. This was in agreement with Hamed et al. and Vural et al. [10-12].

The positive association between clinical outcome and cortical lesions which detected by DIR sequence is considered a great advantage of DIR sequence overcoming the clinico-radiological paradox in MS. The second major advantage of DIR in this study is better differentiation between juxtacortical and leukocortical lesions due to the sharp delineation between white matter and grey matter 


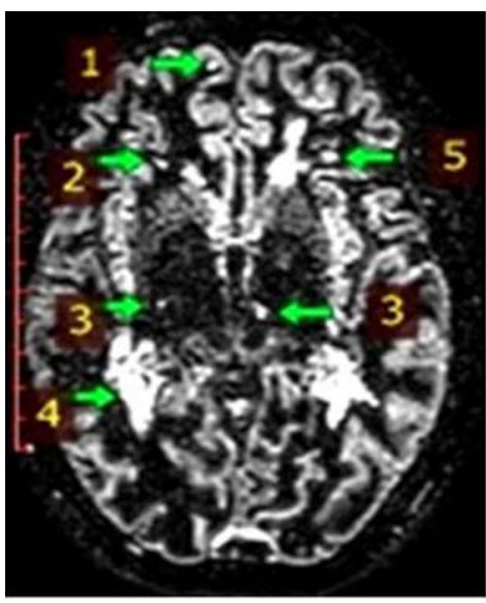

A

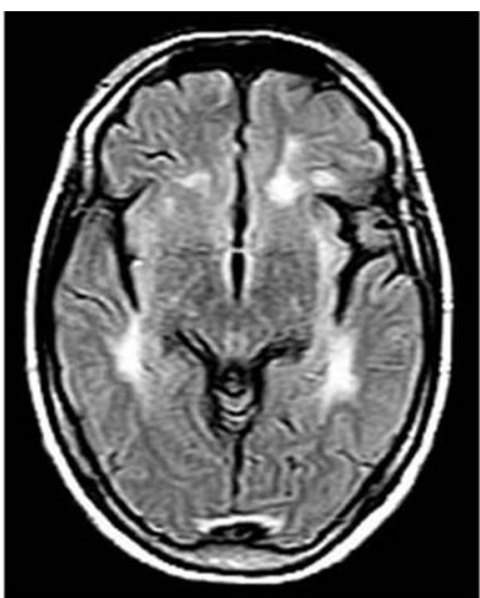

B

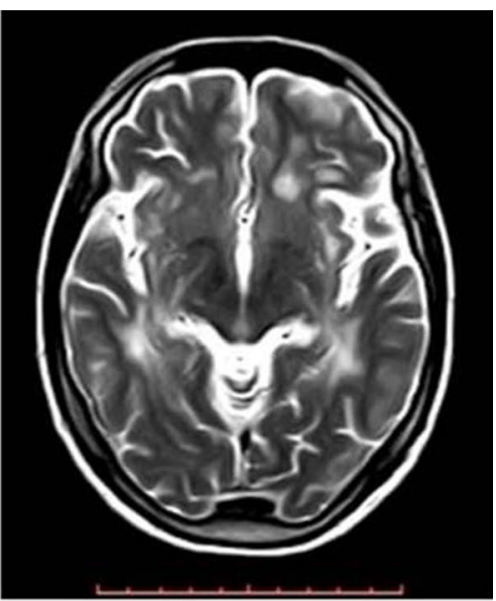

C

Fig. 4 Female patient 32-years-old with history of RRMS since 3 years presented by weakness, headache and visual disorder, EDSS $=4$. A-C (Axial DIR, Flair and T2 sequences, respectively) show MS plaques ( $1=$ juxtacortical lesion, 2 =WM lesion, 3 = lesions at deep grey matter (involving both thalami), $4=$ confluent lesion, $5=$ WM Lesion)

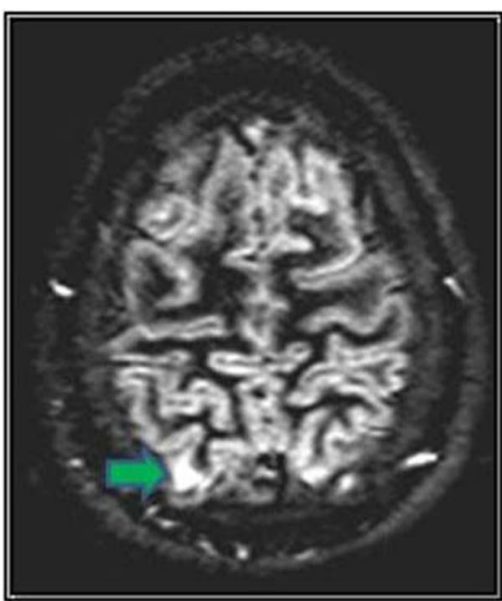

A

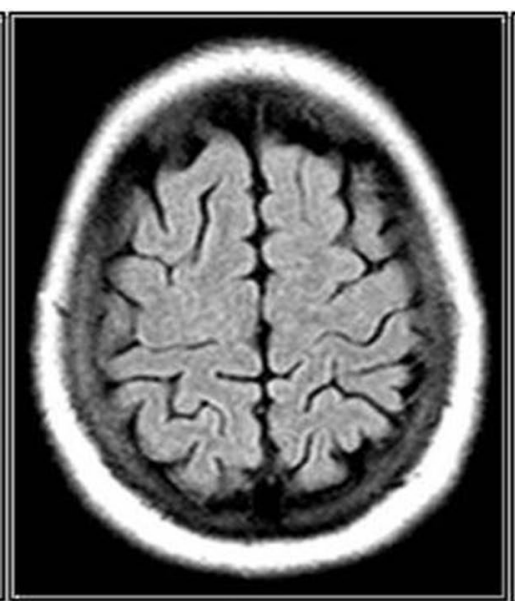

B

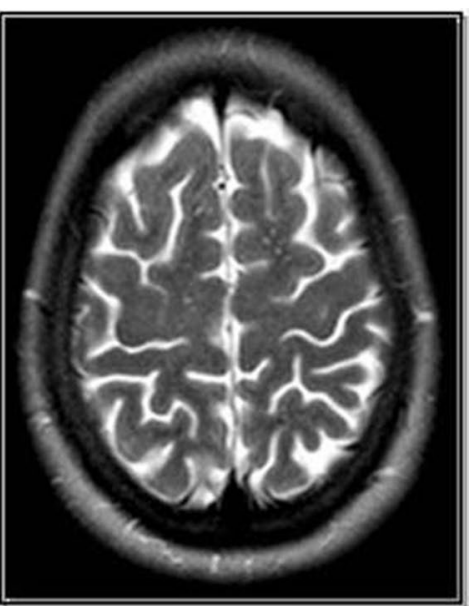

C

Fig. 5 Female patient 27-years-old with history of RRMS since 1 year presented by headache, EDSS =2. A-C (Axial DIR, Flair and T2 sequences, respectively) show the arrow points to an abnormal high signal intensity intra-cortical lesion visualized at the right posterior high parietal level seen at DIR sequence and not visualized at other sequences

Table 1 Socio-demographic data among 40 MS patients:

\begin{tabular}{lll}
\hline Variable & \multicolumn{2}{l}{$\begin{array}{l}\text { Descriptive statistics } \\
\boldsymbol{N}=\mathbf{4 0}\end{array}$} \\
\cline { 2 - 3 } & Mean \pm SD & Range \\
\hline Age (years) & $29.2 \pm 7.1$ & $20-47$ \\
Sex & Female & $35(87.5 \%)$ \\
& Male & $5(12.5 \%)$ \\
\hline
\end{tabular}

Table 2 Socio-demographic data among 20 controls:

\begin{tabular}{lll}
\hline Variable & $\begin{array}{l}\text { Descriptive statistics } \\
\boldsymbol{N}=\mathbf{2 0}\end{array}$ \\
\cline { 2 - 3 } & Mean \pm SD & Range \\
\hline Age (years) & $32.75 \pm 9.23$ & $24-48$ \\
Sex & Female & $20(100 \%)$ \\
& Male & $0(0 \%)$ \\
\hline
\end{tabular}


Table 3 Percentage of the distribution of multiple sclerosis plaques in different anatomical locations of the brain among studied MS patients

\begin{tabular}{llr}
\hline Variable & $\begin{array}{l}\text { Distribution of plaques } \\
\text { in different anatomical } \\
\text { locations of the brain } \\
\text { among } 40 \text { MS patients }\end{array}$ \\
\cline { 2 - 3 } & N & $\%$ \\
\hline Leukocortical region & 22 & 55 \\
Intracortical region & 10 & 25 \\
\hline
\end{tabular}

in DIR as some lesions which are scored on FLAIR and T2 images as juxtacortical lesions often turned out to be leukocortical (mixed white matter grey matter lesions) on DIR sequence.

The converged results between DIR and FLAIR sequences return to CSF signal nulling in both sequences. However, DIR sequence adds better morphological characterization and delineation of the lesions, this is in agreement with Abdullah et al. [13]. So in this study, these lesions become more countable and obvious in DIR sequence compared to the confluent configuration of the lesions in FLAIR and T2 sequences. In addition, in this study DIR sequence adds better morphological characterization and delineation of deep grey matter lesions in comparison with other sequences.
Table 6 Spearmen correlation between number of cortical grey matter lesions in DIR, T2 and Flair sequences versus EDSS score among studied 40 MS patients

\begin{tabular}{lrr}
\hline Spearman correlation & \multicolumn{2}{l}{ EDSS score } \\
\cline { 2 - 3 } & \multicolumn{1}{l}{$\boldsymbol{r}$} & \multicolumn{1}{c}{$\boldsymbol{P}$ value } \\
\hline $\begin{array}{l}\text { Number of cortical grey matter lesions in Flair } \\
\text { sequence }\end{array}$ & 0.896 & $<0.001^{*}$ \\
Number of cortical grey matter lesions in DIR sequence & 0.912 & $<0.001^{*}$ \\
Number of cortical grey matter lesions in T2 sequence & 0.788 & $0.012^{*}$ \\
\hline Significant level at $P$ value $<0.05$ & &
\end{tabular}

The importance of grey matter damage in MS patients and its effect on patient's disability as well as the need for more assessment of disease progression makes visualization and quantification of cortical lesions on routine scan is a priority and excites us to introduce DIR sequence in our routine study.

\section{Conclusion}

Grey matter brain damage is common in multiple sclerosis (MS) so changes the classic concept of this disease as a pure white matter disease. Double inversion recovery (DIR) is 1.5-5 times more sensitive than conventional MRI in detection of the grey matter lesions especially cortical lesions. So DIR is a highly recommended sequence to be used in routine MRI protocols for MS study.

Table 4 Comparison between different MRI pulse sequences (T2, Flair and DIR) as regarding detection rate and number of cortical grey matter lesions among studied 40 MS patients:

\begin{tabular}{llll}
\hline Variable & $\begin{array}{l}\text { T2 } \\
\text { sequence }\end{array}$ & $\begin{array}{l}\text { Flair } \\
\text { sequence }\end{array}$ & $\begin{array}{l}\text { DIR } \\
\text { sequence }\end{array}$ \\
\hline $\begin{array}{l}\text { Detection rate of cortical grey matter lesions (intra- } \\
\text { cortical and leukocortical) }\end{array}$ & $9(22.5 \%)$ & $20(50 \%)$ & $24(60 \%)$ \\
Number of cortical grey matter lesions & $\begin{array}{l}(6-11) \wedge \\
8 \pm 1.7 \wedge \wedge\end{array}$ & $\begin{array}{l}(2-20) \wedge \\
10.7 \pm 5.2 \wedge \wedge\end{array}$ & $\begin{array}{l}(1-22) \wedge \\
10.6 \pm 6.5 \wedge \wedge\end{array}$ \\
\hline
\end{tabular}

$\wedge$ Range, $\wedge \wedge$ Mean \pm SD

Repeated measures ANOVA test

Significant level at $P$ value $<0.05$

Table 5 Agreement between DIR, T2 and Flair results as regarding diagnostic accuracy parameters among studied 40 MS patients

\begin{tabular}{|c|c|c|c|c|c|c|}
\hline Variable & DIR & Flair & $\mathrm{T} 2$ & $\begin{array}{l}\text { DIR versus Flair } \\
\text { (Kappa) } \\
P \text { value }\end{array}$ & $\begin{array}{l}\text { DIR versus T2 } \\
\text { (Kappa) } \\
P \text { value }\end{array}$ & $\begin{array}{l}\text { Flair versus T2 } \\
\text { (Kappa) } \\
P \text { value }\end{array}$ \\
\hline \multicolumn{7}{|c|}{ Cortical grey matter lesions } \\
\hline $\begin{array}{l}\text { No } \\
\text { Yes }\end{array}$ & $\begin{array}{l}16(40 \%) \\
24(60 \%)\end{array}$ & $\begin{array}{l}20(50 \%) \\
20(50 \%)\end{array}$ & $\begin{array}{l}31(77.5 \%) \\
9(22.5 \%)\end{array}$ & $\begin{array}{l}(0.8) \\
<0.001^{*}\end{array}$ & $\begin{array}{l}(0.32) \\
0.005^{*}\end{array}$ & $\begin{array}{l}(0.52) \\
<0.001 *\end{array}$ \\
\hline
\end{tabular}

Significant level at $P$ value $<0.05$ 
Table 7 Comparison between different MRI sequences (T2, Flair and DIR) among studied 40 MS patients versus controls as regarding detection rate of cortical grey matter lesions

\begin{tabular}{llllllr}
\hline Variable & DIR & Flair & T2 & Control & & \multicolumn{2}{l}{ P value versus Control } \\
\cline { 5 - 6 } & & & & DIR & FLAIR \\
\hline Cortical greymatterlesions & & & & $<0.001^{*}$ & $<0.001^{*}$ \\
No & $16(40 \%)$ & $20(50 \%)$ & $31(77.5 \%)$ & $20(100 \%)$ & $0.023^{*}$ \\
Yes & $24(60 \%)$ & $20(50 \%)$ & $9(22.5 \%)$ & $0(0 \%)$ & \\
\hline
\end{tabular}

Significant level at $P$ value $<0.0$

Table 8 ROC curve analysis for the prediction of cortical grey matter lesions using different MRI pulse sequences (DIR, Flair and T2) among studied 40 MS patients (control group is the reference group)

\begin{tabular}{llll}
\hline & DIR & Flair & T2 \\
\hline AUC & 0.8 & 0.75 & 0.613 \\
$95 \% \mathrm{Cl}$ & $0.69-0.91$ & $0.63-0.87$ & $0.47-0.76$ \\
P value & $<0.001^{*}$ & $0.002^{*}$ & 0.158 \\
Sensitivity & 60 & 50 & 22.5 \\
Specificity & 100 & 100 & 100 \\
PPV & 100 & 100 & 100 \\
NPV & 55.6 & 50 & 39.2 \\
Accuracy & 73.3 & 66.7 & 48.3 \\
\hline
\end{tabular}

*Significant $p$-value

\section{Abbreviations}

MS: Multiple sclerosis; DIR: Double inversion recover; EDSS: Expanded disability status scale; MRI: Magnetic resonance imaging; CSF: Cerebrospinal fluid; ICLs: Intra-cortical lesions; LCLs: Leukocortical lesions; CIS: Clinically isolated syndrome; FLAIR: Fluid attenuation inversion recovery; TR: Time of repetition; TE: Time of echo; NSA: Number of signal averages; FOV: Field of view; PPV: Positive predictive value; NPV: Negative predictive value; UC: Area under curve; ROC: Receiver operating characteristic curve; CNS: Central nervous system; RRMS: Relapsing remitting multiple sclerosis; WM: White matter.

\section{Acknowledgements}

$$
\text { Not applicable. }
$$

\section{Authors' contributions}

All authors have appraised the article and actively contributed to the work. $\mathrm{MMH}$ contributed to the idea, image revision and final editing. HSA contributed to the main imaging evaluation and revision. AW and MMI contributed to the data collection and editing. MFA contributed to the main imaging evaluation and revision. All authors read and approved the final manuscript.

\section{Funding}

No sources of funding.

\section{Availability of data and materials}

The datasets used and/or analyzed during the current study are available for the corresponding author on reasonable request.

\section{Declarations}

\section{Ethics approval and consent to participate}

This study was approved by the research ethics committee of Faculty of medicine, at Minia University in Egypt (reference number not applicable). All patients in this study gave a written consent to participate in this research project.

\section{Consent for publication}

Written consent is given to publish data contained in this study.

\section{Competing interests}

The authors declare that they have no competing interests.

\section{Author details}

${ }^{1}$ Department of Radiology, Faculty of Medicine, Minia University, Minya, Egypt. ${ }^{2}$ Department of Neurology, Faculty of Medicine, Minia University, Minya,

Egypt.

Received: 7 July 2021 Accepted: 21 November 2021

Published online: 04 January 2022

\section{References}

1. Geurts JJ, Bö L, Pouwels PJ, Castelijns JA, Polman CH, Barkhof F (2005) Cortical lesions in multiple sclerosis: combined postmortem MR imaging and histopathology. AJNR Am J Neuroradiol 26(3):572-577

2. Geisseler O, Pflugshaupt T, Bezzola L, Reuter K, Weller D, Schuknecht B, Brugger P, Linnebank M (2016) The relevance of cortical lesions in patients with multiple sclerosis. BMC Neurol 16(1):204. https://doi.org/10. 1186/s12883-016-0718-9

3. Nielsen AS, Kinkel RP, Madigan N, Tinelli E, Benner T, Mainero C (2013) Contribution of cortical lesion subtypes at 7T MRI to physical and cognitive performance in MS. Neurology 81:64149. https://doi.org/10.1212/ WNL.0b013e3182a08ce8pmid:23864311

4. Forslin Y, Bergendal $\AA$, Hashim F, Martola J, Shams S, Wiberg MK, Fredrikson S, Granberg T (2018) Detection of leukocortical lesions in multiple sclerosis and their association with physical and cognitive impairment: a comparison of conventional and synthetic phase-sensitive inversion recovery MRI. Am J Neuroradiol. https://doi.org/10.3174/ajnr.A5815

5. Madelin G, Oesingmann N, Inglese M (2010) Double inversion recovery MRI with fat suppression at 7 Tesla: initial experience. J Neuroimag 20:87-92

6. Abdelaziz ME, May AK, Amr ME, Mahmoud LE. Added value of double inversion recovery magnetic resonance sequence in detection of cortical and white matter brain lesions in multiple sclerosis. Department of Radio-diagnosis and Neurology, Faculty of Medicine, Alexandria University, Egypt. Egypt J Radiol Nucl Med. 2014; 45(4), 1193-9.

7. Calabrese M, De Stefano N, Atzori M, Bernardi V, Mattisi I, Barachino L, Morra A, Rinaldi L, Romualdi C, Perini P, Battistin L, Gallo P (2007) Detection of cortical inflammatory lesions by double inversion recovery magnetic resonance imaging in patients with multiple sclerosis. Arch Neurol 64(10):1416-1422. https://doi.org/10.1001/archneur.64.10.1416

8. Filippi M, Preziosa P, Banwell BL, Barkhof F, Ciccarelli O, De Stefano N, Geurts J, Paul F, Reich DS, Toosy AT, Traboulsee A, Wattjes MP, Yousry TA, Gass A, Lubetzki C, Weinshenker BG, Rocca MA (2019) Assessment of lesions on magnetic resonance imaging in multiple sclerosis: practical guidelines. Brain J Neurol 142(7):1858-1875

9. Shilpa K, Makarand M, Aparna P and Apurva W. Role of double inversion recovery sequence in neuro-imaging on 3 Tesla MRI. 2021;69(2): 394-6.

10. Hamed W, Fathi W, Mahmoud W, Elhawary G (2019) Diagnostic accuracy of double inversion recovery in delineation of multiple sclerosis lesions 
and its clinical correlation with expanded disability scoring system. Egypt J Radiol Nucl Med 50:114

11. Abidi Z, Faeghi F, Mardanshahi Z, Mortazavi H (2017) Assessment of the diagnostic accuracy of double inversion recovery sequence compared with FLAIR and T2W_TSE in detection of cerebral multiple sclerosis lesions. Electron Phys 9(4):4162-4170

12. Vural G, Keklikoğlu HD, Temel \$, Deniz O, Ercan K (2013) Comparison of double inversion recovery and conventional magnetic resonance brain imaging in patients with multiple sclerosis and relations with disease disability. Neuroradiol J 26(2):133-142

13. Abdullah D., Rozi M., Subapriya S., Hasyma A. Double inversion recovery in evaluation of multiple sclerosis a narrative review. Rev Latinoam Hipertens. 2019; 14(3)

\section{Publisher's Note}

Springer Nature remains neutral with regard to jurisdictional claims in published maps and institutional affiliations.

\section{Submit your manuscript to a SpringerOpen ${ }^{\odot}$ journal and benefit from:}

- Convenient online submission

- Rigorous peer review

- Open access: articles freely available online

- High visibility within the field

- Retaining the copyright to your article

Submit your next manuscript at $\boldsymbol{\nabla}$ springeropen.com 